\title{
Enhancing sensor monitoring of earthfill dams using Model Order Reduction
}

\author{
Christina Nasika*, Pedro Diez*, Pierre Gerard ${ }^{\dagger}$, Thierry J. Massart ${ }^{\dagger}$ and Sergio \\ Zlotnik* $^{*}$ \\ * International Center for Numerical Methods in Engineering (CIMNE) \\ Universidad Politécnica de Cataluña \\ Campus Norte UPC, 08034 Barcelona, Spain \\ e-mail: \{christina.nasika1, pedro.diez, sergio.zlotnik \}@upc.edu \\ $\dagger$ BATir - Building, Architecture \& Town planning \\ Université Libre de Bruxelles \\ Avenue F.D. Roosevelt 50, B-1050 Brussels, Belgium \\ e-mail: \{thmassar, piergera\}@ulb.ac.be
}

\begin{abstract}
Modern infrastructure monitoring technologies are often based on the fast response of a digital model of the asset for real-time predictions and/or control. In the case of earthfill dams, particularly tailings dams, the dynamic design and functioning of the structure can give rise to a need for realtime model calibration and data assimilation for accurate monitoring. However, these techniques suppose many queries on large models and are often too costly to be performed in real-time. To accelerate the model response and make feasible the incorporation of numerical modeling within the monitoring system, we propose using Model Order Reduction techniques in the transient thermohydro-mechanical system.

The scope of this work is to create a model to support a sensor monitoring system of a tailings dam, by receiving sensor data of local pressure and/or displacement measurements in real time, and evaluating the full stress and deformation fields in the body of the structure, as well as predicting the location of the phreatic surface. Such model can be used for the detection hazardous pressurestress states, for gaining insights in the most critical potential failure mechanisms, but also for the optimal design of the sensor network and the overall optimization of the monitoring system.

POD-based model reduction, combined with Discrete Empirical Interpolation (DEIM) is used to tackle the coupled hydro-mechanical, non-linear, transient problem. The model is implemented in the FEniCS computing platform, an open-source platform that automates the solution of Partial Differential Equations with FEM [1]. It is used for data assimilation applications [2], parameter identification (soil mechanical and hydraulic properties) and optimal sensor placement [3]. The efficiency gains in inverse problem solving and the accuracy of the resulting ROM are examined and discussed.
\end{abstract}

\section{REFERENCES}

[1] M. S. Alnaes, J. Blechta, J. Hake, A. Johansson, B. Kehlet, A. Logg, C. Richardson, J. Ring, M. E. Rognes and G. N. Wells The FEniCS Project Version 1.5. Archive of Numerical Software (2015) Vol. 3, No. 100.

[2] A. Quarteroni, A. Manzoni, F. Negri Reduced Basis Methods for Partial Differential Equations. An introduction. Springer (2015) Vol. 92.

[3] A. Nadal, F. Chinesta, P. Diez, F.J. Fuenmayor \& F.D. Denia Real time parameter identification and solution reconstruction from experimental data using the Proper Generalized Decomposition Computer Methods in Applied Mechanics and Engineering (2015) Vol. 296, Pages 113-128. 